\title{
Características de una muestra de niños con sospecha de abuso sexual en un dispositivo especializado andaluz
}

\section{Characteristic of a sample of children with suspicion of sexual abuse in an Andalusian specialized device}

\section{Encarna Maria SÁNCHEZ ESPINOSA* Irene MARTÍN SÁNCHEZ**}

\section{RESUMEN}

Objetivo: Describir las características más relevantes de los casos con sospecha de abuso sexual evaluado por un equipo especializado. Aporta información sobre la fuente de detección, tipologías de abuso, situación administrativo-legal de los niños; características de éstos y sus familias; relación entre víctima y ofensor y características de los ofensores.

Método: Se realizó un análisis retrospectivo de 198 casos evaluados entre los años 1998 y 2001. Se recoge información sobre 250 niños, 198 madres, 350 parejas víctima ofensor y 277 supuestos ofensores. Se extrajo información relativa a 128 variables.

Resultados: La fuente de detección más frecuente fue la madre. Predomina la alegación de abuso sexual intrafamiliar. Algo menos de la mitad de los niños provenian de protección infantil. Un tercio fueron preescolares. Casi la mitad no convivía con su padre biológico. La relación entre la supuesta víctima y el ofensor más frecuente fue la de padre biológico. La conducta abusiva más general fue tocamientos en genitales. Entre los supuestos ofensores predominan los varones de edad comprendida entre 18 y 40 años.

Conclusiones: Se confirma la presencia de varias características señaladas como factores de riesgo por la literatura. Se discute la mayor representación de algunas de estas características y su similitud o diferenciación con las encontradas en niños víctimas de maltrato y /o abuso sexual.

\footnotetext{
* Diputación Provincial de Sevilla

** Equipo Eicas (ADIMA).
} 
Características de una muestra de niños con sospecha de abuso sexual en un dispositivo especializado andaluz

\section{PALABRAS CLAVE}

Abuso sexual alegado, Niño víctima, Familia, Cuidador, Pareja víctima ofensor, Ofensor.

\section{ABSTRACT}

Objective: Describe the characteristics of alleged sexual abuse cases with the assessment of a team of specialists. Provide information about the source of detection, type of abuse, legal-administrative situation of children, characteristics of children and their families, relation between children and offender and characteristics of offenders.

Method: A retrospective analysis was performed of 198 cases assessed between 1998 and 2001. Information was collected covering 250 children, 198 mothers, 350 offenders' victim's couples and 277 offenders. Information was obtained concerning 128 variables.

Results: Mothers were the most frequent source of detection. The allegation of intrafamily sexual abuse prevails. Slightly less than half of the children came from child protection services. A third were at pre-school age when supposed abuse occurred. Almost half no longer lived with their natural father. The most common abusive behaviour consisted of touching of genitals. The most frequent relation between the supposed victim and offender was that of biological father. Among supposed offenders there was a prevalence of males aged between 18 and 40 .

Conclusions: The study confirmed the presence of several characteristics seen as risk factors in the literature. The greater representation of some of these factors is discussed, as are the similarities or differences with those found in child victims of physical/psychological abuse and/or sexual abuse.

\section{KEY WORDS}

Suspects sexual abuse, Victim child, Family, Caretaker, Offenders. 


\section{INTRODUCCIÓN}

Aunque en número limitado, se han realizado investigaciones sobre abuso sexual infantil en nuestro país en la última década. La primera, que sacó a la luz la existencia de este problema social (López, 1994), fue un estudio de prevalencia con adultos que fueron entrevistados sobre sus recuerdos de experiencias abusivas en su infancia. En cambio, los estudios de incidencia existentes son investigaciones sobre maltrato infantil, en las que el abuso sexual es contemplado como una tipología más del maltrato de que pueden ser objeto los niños y se refieren a determinadas comunidades 0 zonas del país (De Paúl, Arruabarrena, Torres y Muñoz, 1995; Gracia y Musitu,1993; Inglés, 1993; Moreno, Jiménez, Oliva, Palacios y Saldaña, 1995; Verdugo, Gutiérrez, Fuertes y Elice 1993) o al conjunto del estado español (Saldaña, Jiménez y Oliva, 1995). En todos ellos la incidencia del abuso sexual fue en torno al 5\% del maltrato detectado.

En las primeras investigaciones, los datos se obtuvieron de los expedientes de protección, lo que hace que el abuso sexual descrito sea el de menores con desprotección asociada, víctimas de abuso sexual intrafamiliar y con familias de nivel socio-económico desfavorecido. Por tanto, no recogen otros tipos de abuso sexual como el extrafamiliar o el entre iguales, ni a aquellos niños víctimas que no han sido objeto de atención por el sistema de protección infantil y que podrían representar a poblaciones más "normalizadas". Asimismo, dada su finalidad más general, no aportan datos específicos del abuso sexual tales como indicadores, conductas abusivas, duración, gravedad, testimonio y lugar, entre otros aspectos de interés. Ante ese estado de la cuestión, resultan de interés estudios específicos, que ayuden a completar la caracterización en nuestro contexto.
No obstante hay que tener presente una limitación común de estos estudios y del que se presenta y es que la mayoría tienen la dificultad de que se basan en casos con distintos grados de certeza o confirmación del diagnóstico de abuso sexual. Realmente es muy dificil establecer cuáles son casos confirmados de abuso, ya que son pocos los signos fisicos determinantes y las reglas para decidir si una declaración de un menor es falsa 0 verdadera varían según los autores (Garrido y Masip, 2004). Por tanto creemos que es más apropiado hablar de abuso sexual "alegado" o supuesto abuso sexual cuando se describen estos casos.

En los últimos años, la investigación sobre el abuso sexual en nuestro país ha aportado resultados de dispositivos de atención especializada a niños supuestas víctimas de abuso sexual. Estas unidades o equipos de evaluación, recogen casos procedentes de diversas fuentes de derivación, pudiendo así captar a niños con diversas situaciones familiares y sociales y con distintos tipos de victimización. Los primeros estudios realizados con fuentes de ese tipo fueron los estudios de Pou et al. $(1996,2002)$ de la Unidad del Hospital Clínico de San Juan de Dios de Barcelona. En esta misma línea se enmarca el que a continuación se presenta.

Los datos de este estudio proceden del dispositivo especializado "Equipo para la investigación de niños supuestas víctimas de abuso sexual" (EICAS) en Sevilla, programa encuadrado en la Asociación Andaluza para la Prevención del Maltrato Infantil (ADIMA) y en la Federación de Asociaciones para la Prevención del Maltrato Infantil (FAPMI), fue subvencionado por el Ministerio de Trabajo y Asuntos Sociales y en la actualidad por la Junta de Andalucía. Este equipo que estaba compuesto por un trabajador social, cinco psicólogos evaluadores y un psicó- 
logo que diseñó y coordinó el programa, recibía casos con sospecha de abuso sexual derivados por el sistema de protección, escuelas, hospitales, juzgados, policía, centros de la mujer y otros. La procedencia geográfica de los casos era de Andalucia occidental y especialmente de Sevilla y su provincia.

El presente trabajo, es parte de un estudio más amplio. Aquí se describen los resultados de caracterización global de la muestra. Los objetivos de este apartado han sido describir: a) procedencia de los casos y su detección; b) composición de la muestra en cuanto a tipos de abuso sexual alegado y situación administrativo-legal; c) características de los niños y sus familias; d) aspectos relativos al propio abuso o relación entre víctima y ofensor y e) características de los supuestos ofensores.

Se espera con ello ampliar la información sobre casos con sospecha de abuso sexual infantil detectado en el contexto andaluz y aportar sugerencias y elementos que ayuden a planificar nuevos estudios y programas en el campo de la prevención, la evaluación y el tratamiento del citado problema.

\section{MÉTODO}

\section{Casos}

Los casos incluidos en esta investigación son peticiones de estudio de niños de 0 a 18 años, con sospecha de abuso sexual, recibidas en el equipo. Cada petición o caso puede incluir a una o más supuestas víctimas. De las 401 peticiones recibidas en el citado equipo desde 1998 hasta septiembre del 2002 se han incluido en esta investigación 198. Se tomó un porcentaje de las peticiones recibidas en cada año, para así poder tener una perspectiva de la demanda recibida en un periodo amplio. El 29\% de los casos incluidos fueron recepcionados en 1998, el $34 \%$ en 1999 , el $24 \%$ en 2000 y el $12.5 \%$ en 2001 . Los casos incluyen a 250 niños supuestas víctimas, 198 madres o figuras cuidadoras, 350 sospechas de abuso o parejas víctima ofensor y 277 supuestos ofensores.

\section{Material}

El material utilizado para la recopilación de los datos fue elaborado para el estudio. Se construyó un Manual de Definición de variables de abuso sexual, con las definiciones operativas de las 128 variables incluidas en el estudio y una Ficha para la Recogida de Datos, compuesta de siete áreas, en cada una de las cuales se recoge la información relevante respecto de una faceta del objeto de estudio. Además se elaboró una clasificación de indicadores de abuso sexual siguiendo la del Manual "Child Sexual Abuse: Intervention and Treatment Issues" (Faller, 1993), del National Center on Child Abuse and Neglect. Al igual que en éste, se ha distinguido entre indicadores en niños menores o mayores de 10 años, de alta 0 baja probabilidad e indicadores conductuales o fisicos.

La selección de variables y de categorías se realizó atendiendo a las señaladas por la literatura, especialmente las consideradas como factores de riesgo. Otros criterios de selección de variables fueron la disponibilidad de información en el expediente y la opinión del equipo investigador sobre la idoneidad de tal o cual variable para describir y contextualizar la población supuesta víctima de abuso en nuestro ámbito geográfico y cultural. Para la organización y tratamiento de los datos se utilizaron los programas Microsoft Access y SPSS en versión 11.5 y otras previas 


\section{Procedimiento}

La información considerada por cada caso procede de los expedientes e incluye la obtenida por el propio equipo en la evaluación y la enviada por las entidades que derivan (SSCC, Juzgado, etc.). Las fichas de recogida de datos para el estudio eran cumplimentadas por miembros del equipo que evaluaba a los niños, siempre que fue posible sobre los propios casos que cada uno había evaluado, siendo todas revisadas por una de las autoras del estudio. La información de cada ficha era vertida a una base de datos, construida al efecto, que en la actualidad sirve para mantener un registro actualizado de los casos atendidos.

\section{Definición, tipos de abuso y figuras consideradas}

Ante la diversidad de definiciones existentes en la literatura (Finkelhor, 1979; Friedrick, Urquiza y Bielke 1986; Leitenberg et al. 1992; López 1995; Rind, Tromovitch y Bauserman,1998; Schaaf y McCann 1988), se consideró como caso con sospecha de abuso, o caso de abuso alegado a aquel cuya petición recibida en el equipo, fue referida a un menor o menores de 18 años con sospecha de haber estado o estar implicado en una interacción de carácter sexual, con o sin contacto físico, que se produjese con asimetría o coerción. La asimetría se ha establecido por una superior capacidad fisica y psíquica del ofensor sobre la víctima, entendida cuando hay al menos cinco años de diferencia entre víctima y ofensor hasta los 16 años y de 10 años a partir de los 16. La coerción se definió como el uso de fuerza física, amenazas o intimidación.

Los tipos de abuso sexual considerados han sido los siguientes:
- Intrafamiliar: La víctima y ofensor forman parte de la misma familia. El ofensor es un miembro adulto de la estructura familiar y tiene una relación de consanguinidad con la víctima de hasta $4^{\circ}$ grado 0 es un adulto sin relación biológica, miembro de la estructura familiar, que representa un rol familiar y convivencial con el niño.

- Extrafamiliar: El ofensor es un adulto, mayor de 18 años, que no tiene lazos de consanguinidad con el menor o éstos no son los descritos como propios de abuso sexual intrafamiliar, no convive con él en el domicilio familiar, ni ocupa un rol de parentesco convivencial.

- Entre iguales: El ofensor tiene menos de 18 años y entre víctima y ofensor existe una diferencia de edad de 50 más años. Si esa diferencia es menor se considera que hay abuso si se trata de una sola víctima y varios ofensores que actúan a la vez agrediendo sexualmente y/o queda probada la existencia de coerción. Se ha distinguido entre casos de intra y extrafamiliar.

- De varias tipologías: Si presenta alguna combinación de dos o más de los anteriores tipos de abuso sexual.

Las figuras contempladas han sido:

Pareja víctima-ofensor o sospecha: Se refiere a un niño supuesta víctima y su supuesto ofensor. Pareja víctima-ofensor o sospecha en el presente estudio se usan como sinónimos y agrupan a las variables que describen la relación entre el niño y el ofensor.

Figura cuidadora/madre: Aquel de los progenitores o persona sustituta que se ocupa fundamentalmente de los cuidados y protección del niño en el momento del abuso. Por cada niño víctima y/o grupo de hermanos se ha estudiado a 
una figura cuidadora o cuidador principal.

\section{RESULTADOS}

\section{LA PROCEDENCIA DE LOS CASOS Y SU DETECCIÓN}

La mayoría de los casos del estudio, concretamente 154 (77.8\%), procedian de Sevilla y su provincia y los restantes $44(22.2 \%)$ fueron derivados de otras provincias de Andalucía Occidental. Respecto a la procedencia geográfica de los menores $107(42.5 \%)$ procedian de capitales de provincia, 80 (31.5\%) de municipios de más de 10.000 habitantes y 59 (23.4\%) de poblaciones de menos de 10.000 habitantes.

La fuente de detección en 92 (46.5\%) casos fue la madre de la supuesta víctima. Si distinguimos entre fuentes no profesionales y profesionales, las no profesionales detectaron $133(67.2 \%)$ casos y las profesionales 63 (31.8\%); no se tuvo esta información en $2(1.0 \%)$ casos. La fuente de derivación profesional con mayor volumen de casos derivados fue protección infantil y servicios sociales comunitarios con 101 (51.0\%), seguidas del ámbito judicial y policial con 54 $(27.3 \%)$, salud con $14(7.1 \%)$ y otras fuentes con 29 (14.6\%) casos.

El primer contacto del equipo evaluador con la fuente de derivación se produjo en un intervalo de 5 días desde la fecha de recepción del caso para 112 (64.4\%) casos, y antes de 15 dias en 145 (83.4\%). Se recibieron una media de 3.77 informes o documentos por caso. Cada niño antes de llegar al equipo había sido entrevistado por una media de 1.29 profesionales. Ásí, 119 (47.6\%) niños no habian sido entrevistados por ningún profesional, 45 (18.0\%) lo fue por uno, y

\section{Gráfico 1. Tipología de abuso en el niño}

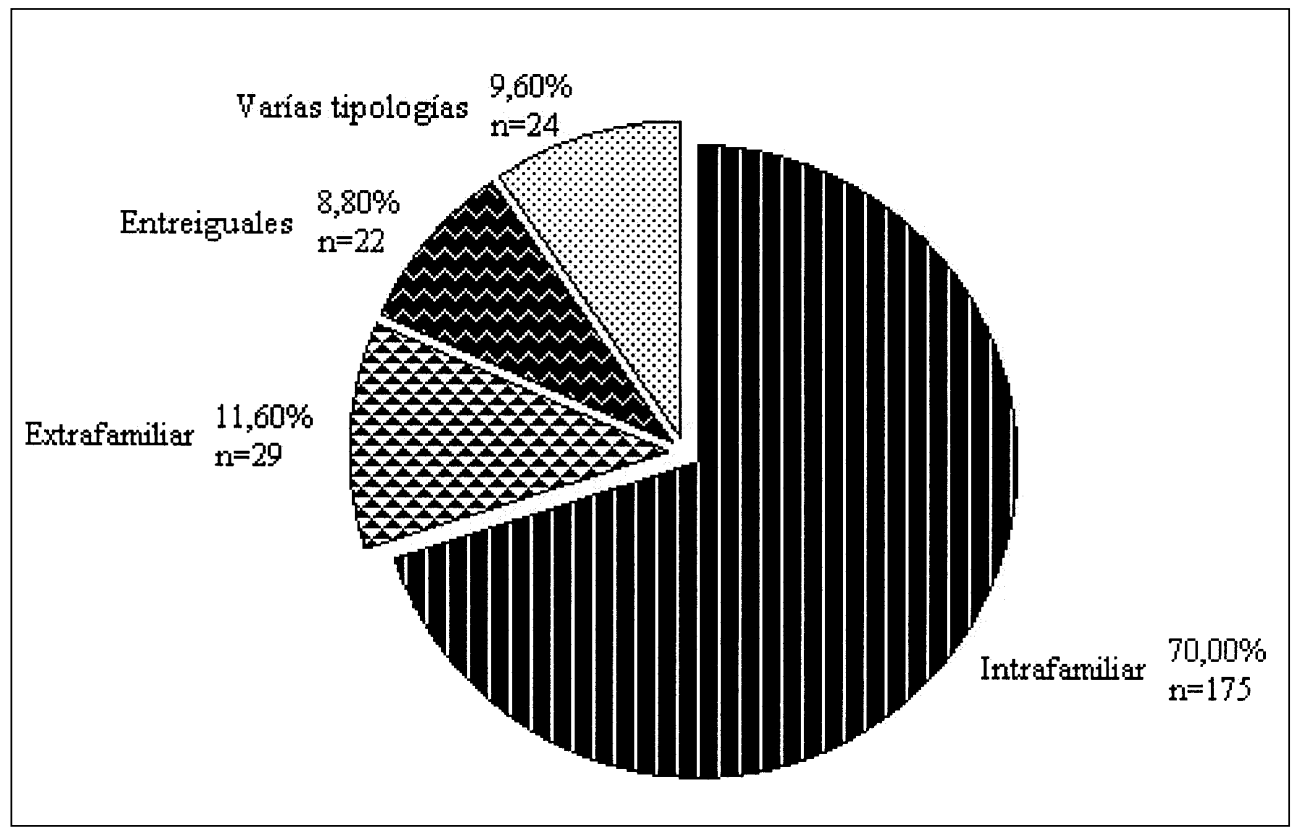


$86(34.4 \%)$ por más de dos. Los tres ámbitos que con mayor frecuencia entrevistaron al menor fueron justicia, que lo hizo en $48(19.2 \%)$ niños, salud en 42 $(17.2 \%)$ y policía, en $32(12.8 \%)$. Los niños más entrevistados fueron los de 13 a 15 años, con una media de 2.05 profesionales, frente a los de 0 a 3 años en los que la media fue de 0.46 .

\section{TIPOS DE ABUSO SEXUAL ALEGADO Y SITUACIÓN ADMINISTRATIVO-LEGAL}

El total de niños supuestas víctimas de abuso sexual en la muestra fue de 250. De ellos $175(70 \%)$ fueron supuestas víctimas de intrafamiliar, de los cuales $146(83.4 \%)$ tenían con el supuesto ofensor una relación de consanguinidad y 29 (16.6\%) sin consanguinidad. Los niños supuestas víctimas de abuso extrafamiliar fueron $29(11.6 \%)$ y de supuesto abuso entre iguales 22 (8.8\%), correspondiendo a entre iguales intrafamiliar $7(2.8 \%)$ y entre iguales extrafamiliar 15 (6.0\%). Los niños supuestas víctimas de varias tipologias fueron 24 (9.6\%)

Como puede verse en la gráfica 1, predomina el abuso sexual intrafamiliar, pero la muestra nos ofrece una diversidad en cuanto a los tipos de abuso sexual del que pueden ser objeto los niños.

La situación administrativo legal fue $66(26.4 \%)$ niños con expediente judicial abierto, 65 (26.0\%) con expediente de protección abierto, 49 (19.6\%) presentaban las dos circunstancias anteriores y $70(28.0 \%)$ no tenian expediente abierto de ningún tipo.

En la gráfica 2 se observan cuatro grupos con un volumen similar de casos. Destaca que algo más de un cuarta parte fueron niños sin ningún

\section{Gráfico 2. Situación administrativa y legal del niño}

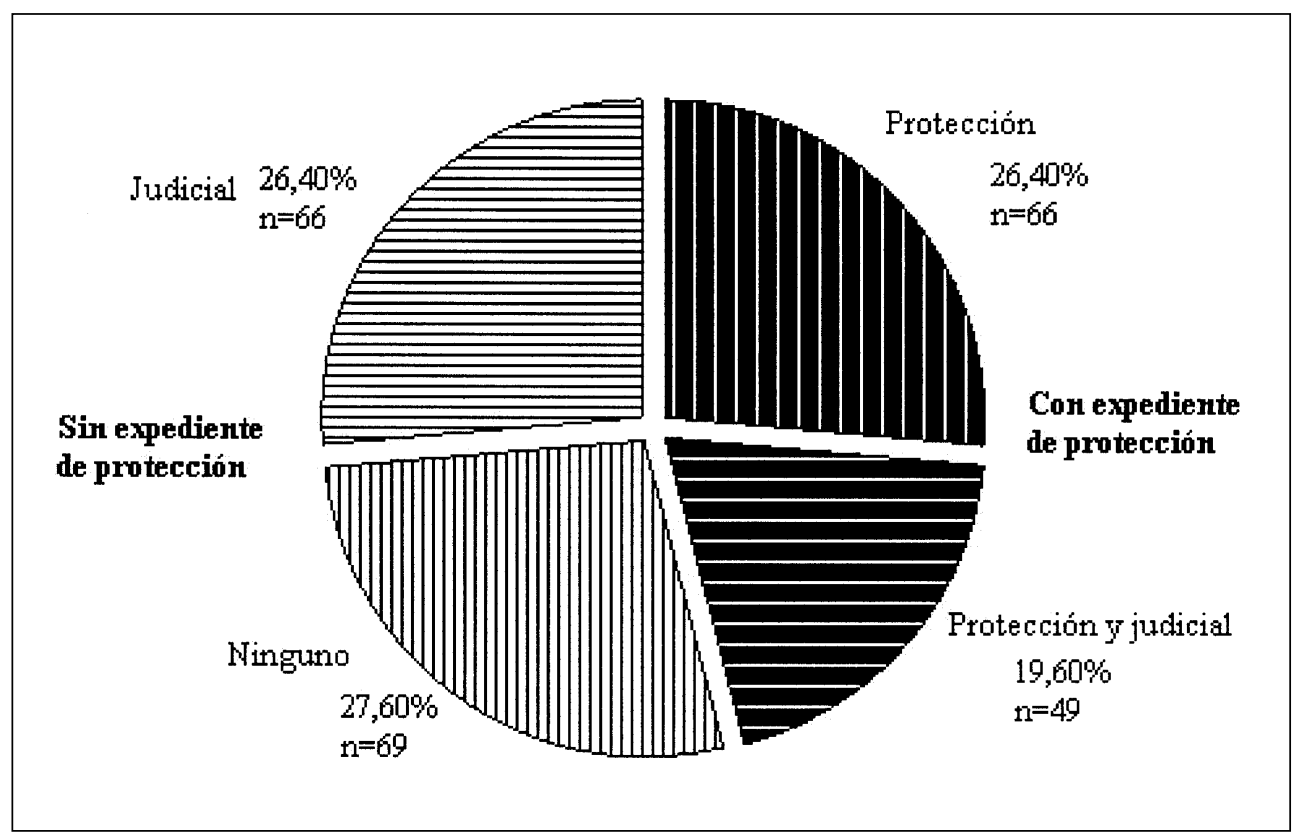


tipo de relación con el sistema judicial ni con el de protección de menores. Atendiendo únicamente a la pertenencia o no del niño al sistema de protección, se observa que el porcentaje de los que no pertenecian al sistema de protección $(54.4 \%)$ fue ligeramente superior al de los que pertenecian $(45.6 \%)$.

\section{CARACTERÍSTICAS DE LOS NIÑOS Y SUS FAMILIAS}

Las características de los niños supuestas víctimas de abuso sexual estudiados se exponen en la tabla 1.

De los anteriores resultados se deduce que hay una proporción de 2.52 niñas por cada niño. Más de la mitad de los niños (58.8\%) tenían menos de 10 años y el rango de edad que más frecuentemente fue derivado para estudio era el de 4 a 6 años. La edad media de los niños fue de 8.8 años. Casi la mitad (48.0\%) tenían antecedentes o presencia de otros maltratos asociados y en algo más de uno de cada cuatro, el supuesto abuso se inició antes de los 6 años. Por otra parte, algo menos de uno de cada cuatro niños presentaba absentismo escolar. Además, el $40 \%$ de los niños fueron explorados médicamente y algo más de uno de cada diez presentaba dêficit físico o psíquico.

\section{Indicadores de abuso sexual}

\section{Indicadores físicos}

Se tuvo constancia de indicadores físicos en 65 (26\%) niños y en 176 (70\%) no, siendo desconocido el dato en 9 (3.6\%) niños. De los que presentaban indicadores, en 49 niños (75.4\%) eran de baja probabilidad, en $5(7.7 \%)$ de alta y en 11 (16.9\%) de ambos tipos. Los dos indicadores físicos de alta probabilidad que se reiteraron fueron: la abertura himineal

Tabla 1. Características niño supuesta víctima

\begin{tabular}{|c|c|c|c|c|c|}
\hline & $\mathbf{N}$ & $\%$ & & $\mathbf{N}$ & $\%$ \\
\hline Género & & & Otros maltratos & & \\
\hline Femenino & 179 & 71.6 & Sí & 120 & 48.0 \\
\hline Masculino & 71 & 28.4 & No & 93 & 37.2 \\
\hline Edad & & & No se conoce & 37 & 14.8 \\
\hline $0-3$ años & 28 & 11.2 & Déficit físico y/o psíquico & & \\
\hline 4-6 años & 73 & 29.2 & Sí & 34 & 13.6 \\
\hline 7-9 años & 46 & 18.4 & No & 189 & 75.6 \\
\hline 10-12 años & 48 & 19.2 & No se conoce & 27 & 10.8 \\
\hline 13-15 años & 40 & 16.0 & Absentismo escolar & & \\
\hline 16-18 años & 12 & 4.8 & Sí & 58 & 23.2 \\
\hline Edad de inicio del abuso & & & No & 160 & 64.0 \\
\hline $0-5$ años & 62 & 24.8 & No se conoce & 32 & 12.8 \\
\hline 6-11 años & 54 & 21.6 & Exploración médica & & \\
\hline 12-18 años & 25 & 10.0 & Sí & 98 & 39.2 \\
\hline No se conoce & 109 & 43.6 & No & 117 & 48.8 \\
\hline & & & No se conoce & 35 & 14.0 \\
\hline
\end{tabular}


agrandada para la edad con signos asociados de ruptura o ausencia de himen, que se dio en 8 (3.2\%) niños, y las lesiones recientes en genitales, ano o paladar sin una explicación accidental adecuada, que se dieron en $5(2.0 \%)$ niños. Los indicadores físicos de baja probabilidad más frecuentes fueron: el eritema vaginal y o perianal que se dio en 37 (14.8\%) niños, dolor en genitales en 19 (7.6\%), prurito en 14 (5.6\%) e infecciones de trasmisión no exclusivamente sexual (vulvovaginitis, vulvitis, vaginitis, candidiasis o herpes entre otras) en 7 (2.8\%) niños, y dilatación anal refleja en 6 $(2.4 \%)$ niños.

\section{Indicadores conductuales}

La presencia de indicadores conductuales fue casi generalizada. Estaban presentes en $220(88.0 \%)$ niños, no se consignaron en $23(9.2 \%)$ y no se tuvo información en 7 (2.8\%). De los que pre- sentaban indicadores $155(70.5 \%)$ presentaron una combinación de indicadores de alta y baja probabilidad, 37 (16.8\%) sólo de alta y 28 niños (12.7\%) sólo de baja.

Los indicadores conductuales de alta y baja probabilidad de mayor frecuencia, encontrados en los 163 (45.2\%) niños pequeños (de hasta 10 años de edad), aparecen en la tabla 2.

La relación de indicadores con mayor presencia en los 87 (34.8\%) niños mayores (más de 10 años) y su frecuencia aparece en la tabla 3.

En las siguientes tablas se observa que el indicador más presente a cualquier edad es la verbalización del niño de que ha sido objeto de abuso sexual. En los pequeños los miedos inespecíficos fueron el siguiente indicador más presente y en los mayores las dificultades en la escuela.

Tabla 2. Indicadores de abuso sexual en niños pequeños ( $<10$ años superior)

\begin{tabular}{|lcc|}
\hline Indicadores conductuales alta probabilidad & N & $\%$ \\
\hline Verbalizar que se es objeto de abuso & 117 & 71.8 \\
Manifestaciones sobre conocimientos sexuales superiores a su edad & 37 & 22.7 \\
Juegos y actividades sexuales con iguales. & 30 & 18.4 \\
Invitaciones sexuales, acciones o comportamientos verbalizados hacia adultos & 25 & 15.3 \\
Masturbación excesiva & 22 & 13.5 \\
Conducta o lenguaje sexualizado & 19 & 11.7 \\
Juegos sexuales con muñecos o animales & 18 & 11.0 \\
\hline Indicadores conductuales baja probabilidad & \multicolumn{3}{|}{} \\
\hline Miedos o Temores & 70 & 42.9 \\
Cambios repentinos en la conducta & 55 & 33.7 \\
Trastornos del sueño & 42 & 25.8 \\
Dificultades en la escuela & 29 & 17.8 \\
Problemas en la relación con los iguales & 28 & 17.2 \\
Enuresis & 23 & 14.1 \\
\hline
\end{tabular}


Tabla 3. Indicadores de abuso sexual en niños mayores ( $\geq 10$ años)

\begin{tabular}{|lcc|}
\hline Indicadores conductuales alta probabilidad & N & $\%$ \\
\hline Verbalizar que se es objeto de abuso & 55 & 63.2 \\
Promiscuidad sexual (especialmente en chicas) & 20 & 23.0 \\
\hline Indicadores conductuales baja probabilidad & & \\
\hline Dificultades en la escuela & 46 & 52.9 \\
Problemas en la relación con los iguales & 42 & 48.3 \\
Cambios repentinos en la conducta & 27 & 31.0 \\
Depresión o Tristeza & 26 & 29.9 \\
Micdo y reticencias hacia el supuesto ofensor & 24 & 27.6 \\
Aislamiento social & 20 & 23.0 \\
Rebeldía & 20 & 23.0 \\
Inversión de roles cn la familia o pscudomadurez & 15 & 17.2 \\
Conductas autodestructivas: automutilaciones e intentos de suicidio & 14 & 16.1 \\
Actividades delictivas & 11 & 12.6 \\
\hline
\end{tabular}

\section{LA FAMILIA DEL NIÑO}

El número de niños supuestas víctimas de abuso en cada una de las 198 familias estudiadas fue de uno sólo en $161(81.3 \%)$ familias, dos en $29(14.6 \%)$, y más de dos en 8 (4.0\%) familias. Varias de las características de las familias aparecen en la tabla 4.

En la tabla 4 se observa que predomina claramente la estructura familiar compuesta por ambos progenitores (69.7\%) aunque destaca el elevado porcentaje de

Tabla 4. Caracteristicas de la familia

\begin{tabular}{|lcc|lcc|}
\hline & $\mathbf{N}$ & $\mathbf{\%}$ & & $\mathbf{N}$ & $\mathbf{\%}$ \\
\hline Estructura familiar & & & Nivel socioeconómico & & \\
Biparental & 138 & 69.7 & Alto / Medio & 74 & 37.4 \\
Monoparental & 42 & 21.2 & Bajo & 74 & 37.4 \\
Extensa & 11 & 5.6 & Precario & 21 & 10.6 \\
No se conoce & 7 & 3.5 & No se conoce & 29 & 14.6 \\
\hline Cambios en la estructura familiar & & Hacinamiento en la familia & \\
Sí & 30 & 15.2 & Sí & 33 & 16.7 \\
No & 156 & 78.8 & No & 122 & 61.6 \\
No se conoce & 12 & 6.1 & No se conoce & 43 & 21.7 \\
\hline Parejas en situación de disputa & & Violencia doméstica & & \\
Sí & 44 & 22.2 & Sí & 94 & 47.0 \\
No & 140 & 70.7 & No & 59 & 29.8 \\
No se conoce & 14 & 7.1 & No se conoce & 46 & 23.2 \\
\hline
\end{tabular}


familias monoparentales (21.2\%). El porcentaje de parejas en disputa $(22.2 \%)$, definidas como aquellas que mantienen en el momento de la exploración del niño un conflicto legal, económico, o emocional es elevado. El desvelamiento del supuesto abuso sexual produjo cambios (por ejemplo, separación de los padres) en la estructura familiar en un $15.2 \%$ de familias. Casi la mitad de las familias presentaron problemas de violencia doméstica. El nivel socioeconómico se ha distribuido con porcentajes similares para el nivel medio/alto de un lado y el bajo y precario de otro. Sin embargo, si agrupamos las categorías de bajo y precario, casi la mitad de las familias estarian en el nivel socioeconómico que podríamos denominar desfavorecido.

\section{Los padres y los hermanos}

Una mayoría de niños, 211 (84.4\%), convivian con su madre biológica (sin que ello excluya la convivencia con otros miembros de la familia), 34 (13.6\%) no convivía, y no se tuvo información en 5 (2.0\%). Con el padre biológico convivian $139(55.6 \%)$ niños y $105(42.0 \%)$ no convivían; no se tuvo información en 6 $(2.4 \%)$ niños. De los niños que no convivian con su padre biológico: 41 (39.0\%) lo hacian con un padre convivencial 0 adoptivo, $60(66.2 \%)$ no convivían con ninguna figura paterna y $17(16.2 \%)$ lo hacian con su familia extensa, no se tuvo esta información en 4 (3.8\%).

Del total de niños supuestas víctimas, el $35.2 \%$ eran hermanos entre sí, que también fueron supuestas víctimas de abuso. De entre los hermanos no supuestas víctimas, la edad media fue 11.6 años. Los hermanos sin antecedentes de maltrato de cualquier tipo, fueron 79 (38.0\%); sí presentaron maltrato 72 (34.6\%) y el dato fue desconocido en 57 $(27.4 \%)$. De los 72 hermanos que pre- sentaron maltrato, hubo antecedentes de abuso sexual en $15(20.8 \%)$ hermanos.

\section{LA FIGURA CUIDADORA DEL NIÑO}

Se estudiaron 198 figuras de cuidado del niño, de las que 174 (87.95\%) eran madres, $5(2.5 \%)$ padres, $12(6.15)$ otros y en $7(3.5 \%)$ no procedía consignar esta figura. Las características estudiadas en este grupo aparecen en la tabla 5.

En la anterior tabla se observa que aproximadamente la mitad de las figuras cuidadoras no tiene ningún tipo de estudios o sólo los estudios primarios, una de cada tres pasaba mucho tiempo fuera del hogar y considerando sólo las que se conoció el dato, fueron la mitad. Una de cada cuatro presentaba consumo abusivo de sustancias, especialmente alcohol, y la misma proporción presentaba algún déficit o trastorno físico o psíquico. Casi la mitad de entre las que se conoció el dato, presentaron antecedentes de maltrato y de éstas la mitad tenían antecedentes de victimización sexual.

La actitud de la figura cuidadora o madre ante el abuso sexual alegado fue valorada en 319 sospechas o parejas víctima ofensor. La actitud de apoyo al niño ocurrió en 145 (45.5\%) sospechas, apoyo al supuesto ofensor en $61(19.1 \%)$, ambivalencia en 54 (16.9\%), no procedía en $26(8.2 \%)$ y no se conoció el dato en $33(10.3 \%)$. Hubo dependencia de la figura de cuidado con respecto al ofensor en 96 $(30.1 \%)$ sospechas, pero 10 más frecuente fue no presentar dependencia, como ocurrió en $147(46.1 \%)$, y no se tuvo información en $76(23.8 \%)$ sospechas. Con respecto al tipo de dependencia, fue más frecuente presentar dependencia emocional (47.9\%) o emocional y económica de modo conjunto (46.9\%). 
Tabla 5. Caracteristicas del cuidador principal

\begin{tabular}{|c|c|c|c|c|c|}
\hline & $\mathbf{N}$ & $\%$ & & $\mathbf{N}$ & $\%$ \\
\hline Edad & & & Estado civil & & \\
\hline $18-30$ años & 51 & 26.7 & Casado & 111 & 58.1 \\
\hline $31-40$ años & 85 & 44.5 & Separado - Divorciado & 54 & 28.3 \\
\hline $41-50$ años & 26 & 13.6 & Soltero & 12 & 6.3 \\
\hline$>50$ años & 10 & 5.2 & Viudo & 6 & 6.1 \\
\hline No se conoce & 19 & 9.9 & No se conoce & 8 & 4.2 \\
\hline Nivel educativo & & & Ocupación & & \\
\hline Sin estudios & 40 & 20.9 & Activo & 84 & 44.0 \\
\hline Estudios primarios & 54 & 28.3 & Desempleado & 70 & 36.6 \\
\hline FP / Bachillerato & 9 & 4.7 & Pensionista / Baja & 11 & 5.8 \\
\hline Estudios superiores & 6 & 3.1 & No se conoce & 26 & 13.6 \\
\hline No se conoce & 82 & 42.9 & \multicolumn{3}{|c|}{ Presencia de trastorno o déficit } \\
\hline Tiempo fuera del hogar & & & Sí & 33 & 17.3 \\
\hline Sí & 60 & 30.3 & No & 104 & 54.5 \\
\hline No & 66 & 33.3 & No se conoce & 54 & 28.3 \\
\hline No se conoce & 64 & 32.3 & \multirow{6}{*}{$\begin{array}{l}\text { Antecedentes de maltrato } \\
\text { Sí } \\
\text { No } \\
\text { No se conoce }\end{array}$} & & \\
\hline No procede & 8 & 4.0 & & 36 & 18.8 \\
\hline Abuso de drogas / Alcohol & & & & 46 & 24.1 \\
\hline Sí & 23 & 12.0 & & 109 & 57.1 \\
\hline No & 72 & 37.7 & & & \\
\hline No se conoce & 96 & 50.3 & & & \\
\hline
\end{tabular}

\section{LA SOSPECHA DE ABUSO. LA RELACIÓN NIÑ̃O-OFENSOR}

En cuanto al parentesco entre el supuesto ofensor y el niño, la relación que destaca en primer lugar fue la de figura paterna biológica en 117 (33.8\%) sospechas. El supuesto ofensor fue tío/a del niño en 40 (11.6\%) sospechas, padre/madre no biológico (adoptivo o convivencial) en 29 (8.4\%), pareja del padre/madre en $10(2.9 \%)$, hermano en $25(7.2 \%)$, abuelo en $22(6.4 \%)$, madre biológica en $10(2.9 \%)$ y en $21(6 \%)$ otros familiares. Sin parentesco familiar pero sí conocido del niño hubo $70(20.2 \%)$ sospechas, y no se tuvo información al respecto en $2(0.6 \%)$.
La media de conductas abusivas por sospecha de abuso sexual alegado fue de 1.91. Las conductas abusivas alegadas con mayor representación aparecen en la tabla 6 .

Como se observa en la tabla 6 la conducta abusiva más frecuentemente alegada casi para la mitad de los niños fueron los tocamientos en genitales.

En 235 (67.9\%) sospechas o parejas niño-ofensor hubo revelación del abuso por parte del niño y en $108(31.2 \%)$ no la hubo, desconociéndose el dato en $3(0.9 \%)$ más. Cuando la revelación del abuso se produjo, en $122(51.9 \%)$ sospechas los niños revelaron antes y durante la explo- 
Tabla 6. Conductas abusivas más frecuentes

\begin{tabular}{|lcc|}
\hline Conductas abusivas más frecuentes & $\mathbf{N}$ & $\mathbf{\%}$ \\
\hline Tocamientos del ofensor a la víctima en órganos sexuales por debajo ropa & 158 & 45.7 \\
Obligar a la víctima a realizar tocamientos en genitales ofensor & 93 & 26.9 \\
Exhibicionismo o Voyerismo & 69 & 19.9 \\
Sexo oral & 69 & 19.9 \\
Tocamientos del abusador a la víctima en órganos sexuales por encima ropa & 52 & 15.0 \\
Penetración con pene anal y/o vaginal & 49 & 14.2 \\
Frotamiento de genitales & 40 & 11.6 \\
\hline
\end{tabular}

ración, 89 (37.9\%) previamente y sólo durante la exploración en 24 (10.2\%).

A continuación se presentan los resultados sobre condiciones y circunstancias de la sospecha de abuso alegado.

Como muestra la tabla 7, la duración del abuso alegado larga o crónica (superior a los tres meses 0 un año respectivamente) fue la más frecuente, seguida de los abusos alegados de duración corta (inferior a un mes); la duración media (superior a un mes e inferior a tres meses) fue poco frecuente. En una de cada tres sospechas el acceso del supuesto ofensor al niño es alto. El método preferido por el ofensor para controlar la conducta del niño es la seducción o el soborno. En casi la mitad de los casos, los abusos alegados se produjeron en el domicilio del ofensor.

Gráfico 3. Revelación del abuso

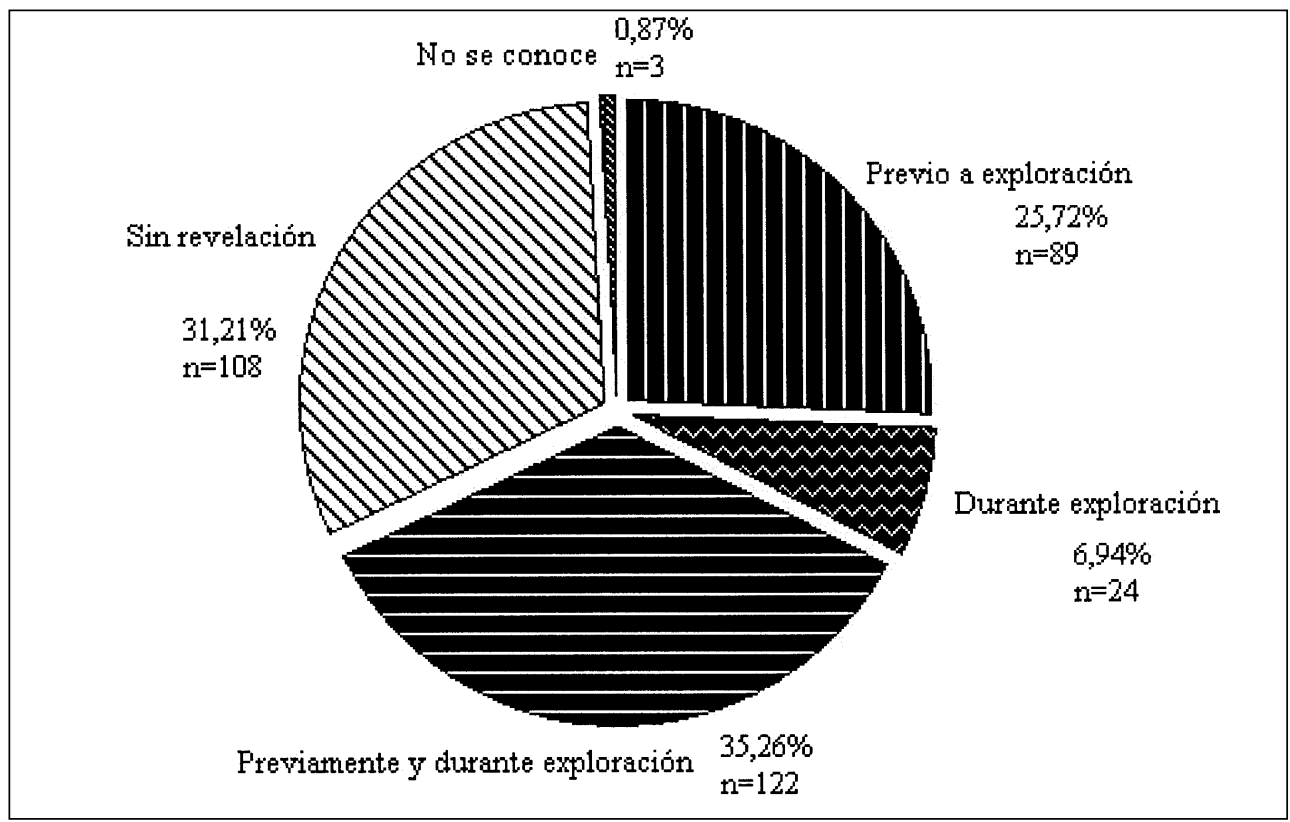


Características de una muestra de niños con sospecha de abuso sexual en un dispositivo especializado andaluz

Tabla 7. Características de la sospecha de abuso

\begin{tabular}{|c|c|c|c|c|c|}
\hline & $\mathbf{N}$ & $\%$ & & $\mathbf{N}$ & $\%$ \\
\hline Duración del abuso & & & Valoración testimonio & & \\
\hline Hasta 1 mes & 70 & 20.2 & Veraz-probablemente & 132 & 38.2 \\
\hline De 1 a 3 meses & 22 & 6.4 & Dudoso & 10 & 2.9 \\
\hline Más de 1 mes & 125 & 36.1 & No veraz & 4 & 1.2 \\
\hline No se conoce & 129 & 37.3 & Sin valoración & 94 & 27.2 \\
\hline \multicolumn{3}{|l|}{ Acceso al menor } & Sin testimonio & 106 & 30.6 \\
\hline Alto & 258 & 74.6 & \multicolumn{3}{|l|}{ Gravedad del abuso } \\
\hline Bajo & 55 & 15.9 & Leve & 54 & 15.6 \\
\hline No se conoce & 33 & 9.5 & \multirow{3}{*}{$\begin{array}{l}\text { Grave } \\
\text { Muy grave } \\
\text { No se estableció }\end{array}$} & 90 & 26.0 \\
\hline Modos empleados & & & & 66 & 19.1 \\
\hline Seducción o sobornos & 115 & 33.2 & & 136 & 39.3 \\
\hline Fuerza física o amenazas & 58 & 16.8 & \multirow{4}{*}{$\begin{array}{l}\text { Diagnóstico del caso } \\
\text { Alta probabilidad } \\
\text { Baja probabilidad } \\
\text { Sin datos concluyentes }\end{array}$} & & \\
\hline Ambas & 53 & 15.3 & & 136 & 39.3 \\
\hline No se conoce & 120 & 34.7 & & 40 & 11.6 \\
\hline Lugar de ocurrencia & & & & 81 & 23.4 \\
\hline Domicilio del menor & 150 & 43.4 & \multirow{4}{*}{$\begin{array}{l}\text { No evaluable - } \\
\text { Sin diagnóstico }\end{array}$} & 89 & 25.7 \\
\hline Domicilio del ofensor & 98 & 28.3 & & & \\
\hline Lugares diferentes & 62 & 17.9 & & & \\
\hline No se conoce & 36 & 10.4 & & & \\
\hline
\end{tabular}

En cuanto a los resultados de la valoración del testimonio del niño, realizada en el equipo por medio de el Análisis del contenido basado en criterios CBCA (Raskin y Esplin 1991) destaca junto a las sospechas con testimonio veraz-probablemente veraz, el grupo clasificado sin testimonio (30.6\%), refiriéndose a declaraciones en las que no hay suficiente producción verbal del niño para realizarla, bien porque no habló, fue escasa, o no evaluable por afectación sensorial o psíquica del niño. También fue elevado el grupo que por diversos motivos no concluye la evaluación (27.2\%). Hubo una presencia escasa de casos con testimonio dudoso o no veraz.

Con respecto al diagnostico final que otorgaba el equipo a cada sospecha estudiada, los resultados presentan como grupo más numeroso el de alta probabilidad. Son sospechas con el nivel de confirmación más alto a juicio de los evaluadores, bien por los resultados de la evaluación de la declaración del niño, bien por la presencia de indicadores fisicos de certeza, o alguna de las dos anteriores circunstancias unidas a indicadores conductuales de alta probabilidad. La baja probabilidad se refiere a sospechas que a juicio del examinador tienen poca evidencia a favor de que el abuso se haya producido. El grupo de sin datos concluyentes engloba a sospechas que a juicio del evaluador eran dudosas, no hay información suficiente para concluir si hubo o no abuso.

Destaca el dato de que casi la mitad de las sospechas se estimaron graves o muy graves. 


\section{CARACTERÍSTICAS DE LOS SUPUESTOS OFENSORES}

Se recogieron datos relativos a 277 supuestos ofensores sexuales. De ese total $220(80.6 \%)$ supuestamente abusaron cada uno de un único niño del estudio, mientras que $43(15.8 \%)$ de dos niños y $10(3.7 \%)$ de más de dos. Además, en 32 (11.7\%) de ellos hubo constancia de denuncia o sospecha fundada de agresiones 0 abusos sexuales previos a otros niños. Es importante resaltar que $5(1.8 \%)$ ofensores fueron niños que tam- bién eran supuestas víctimas en el estudio.

Las características más relevantes se exponen en la tabla 8 , si bien hay que tener en cuenta en algunas variables el alto porcentaje de sujetos de los que no se tuvo acceso a la información prevista.

En la tabla 8 se destaca que predomina el género varón, de edad entre $18 \mathrm{y}$ 40 años, con preferencia por el modo de actuación individual. En cuanto al estado civil es similar el porcentaje de casa-

Tabla 8. Caracteristicas de los supuestos ofensores

\begin{tabular}{|c|c|c|c|c|c|}
\hline & $\mathbf{N}$ & $\%$ & & $\mathbf{N}$ & $\%$ \\
\hline Género & & & \multicolumn{3}{|l|}{ Edad } \\
\hline Varón & 252 & 92.3 & \multicolumn{3}{|l|}{$<18$ años } \\
\hline Mujer & 19 & 7.0 & \multirow{4}{*}{$\begin{array}{l}18 \text { - } 40 \text { años } \\
41 \text { - } 60 \text { años } \\
>60 \text { años } \\
\text { No se conoce }\end{array}$} & \multirow{2}{*}{$\begin{array}{c}105 \\
48\end{array}$} & 38.5 \\
\hline No se conoce & 2 & 0.7 & & & 17.6 \\
\hline Modo de actuación & & & & 21 & 7.7 \\
\hline Individual & 185 & 67.8 & & 48 & 17.6 \\
\hline Díada & 20 & 7.3 & \multicolumn{3}{|c|}{ Antecedentes de maltrato } \\
\hline Grupal & 39 & 14.3 & Sí & 30 & 11.0 \\
\hline No se conoce & 29 & 10.6 & \multirow{2}{*}{$\begin{array}{l}\text { No } \\
\text { No se conoce }\end{array}$} & 20 & 7.3 \\
\hline Nivel educativo & & & & 223 & 81.7 \\
\hline Sin estudios & 35 & 12.8 & \multicolumn{3}{|l|}{ Ocupación } \\
\hline Estudios primarios & 81 & 29.7 & Activo & 89 & 32.6 \\
\hline FP / Bachillerato & 14 & 5.1 & \multirow{2}{*}{ Desempleado } & 35 & 12.8 \\
\hline Estudios superiores & 12 & 4.4 & & 29 & 10.6 \\
\hline No se conoce & 131 & 48.0 & $\begin{array}{l}\text { Pensionista / Baja } \\
\text { Ama de casa }\end{array}$ & 2 & 0.7 \\
\hline \multicolumn{3}{|l|}{ Estado civil } & \multirow{2}{*}{$\begin{array}{l}\text { No se conoce } \\
\text { No procede (escolares) }\end{array}$} & 68 & 24.9 \\
\hline Casado & 91 & 33.3 & & 50 & 18.3 \\
\hline Separado - Divorciado & 98 & 35.9 & \multicolumn{3}{|c|}{ Presencia de trastorno o déficit } \\
\hline Soltero & 33 & 12.1 & \multirow{6}{*}{$\begin{array}{l}\text { Sí } \\
\text { No } \\
\text { No se conoce }\end{array}$} & 25 & 9.2 \\
\hline Viudo & 5 & 1.8 & & 96 & 35.2 \\
\hline No se conoce & 46 & 16.6 & & 152 & 55.7 \\
\hline Abuso de drogas / Alc & & & & & \\
\hline $\begin{array}{l}\text { Sí } \\
\text { No }\end{array}$ & 42 & $\begin{array}{l}26.0 \\
15.4\end{array}$ & & & \\
\hline $\begin{array}{l}\text { No } \\
\text { No se conoce }\end{array}$ & 160 & 58.6 & & & \\
\hline
\end{tabular}


dos y separados o divorciados. El consumo de drogas o alcohol se conoció sólo en algo menos de la mitad siendo predominante entre éstos la presencia de consumo.

\section{DISCUSIÓN}

\section{Caracteristicas de los niños}

En la muestra el tipo de abuso sexual alegado más frecuente ha sido el intrafamiliar, al igual que en los estudios de nuestro país, cuyas muestras son de niños del sistema de protección. Sobre esta cuestión, frecuencia de los tipos de abuso, la literatura ofrece distintos datos: Así, se ha señalado que el intrafamiliar está en torno a la mitad del total de los casos detectados (Finkelhor, 1986), que oscila entre el 10\% y el 50\% (Bagley \& King, 1990), que presenta una ligera diferencia a favor del extrafamiliar (Fisher \& McDonald, 1998), o inclusive que las características de los casos denunciados varian según las fuentes información (Berliner y Elliott, 1996).

En cuanto a las características encontradas en los niños, se confirma el tópico con respecto al genero ya que predominan claramente las niñas, en proporción similar a la de otros estudios en nuestro medio (Pou et al. 2002 y Sanmartín, 2002). El resultado apoya la consideración de Boney-McCoy y Finkelhor (1995) de que ser mujer incrementa el riesgo de padecer abuso sexual. Respecto a la edad, el porcentaje de niños pequeños es mayor al encontrado en el estudio de maltrato en Andalucía (Moreno et al. 1995), más parecido al resultado de nivel estatal (Sanmartín, 2002); y similar a ambos en la notoria disminución del porcentaje de víctimas en el rango de 16 a 18 años.
Casi la mitad de los niños tuvieron antecedentes o presencia de otros tipos de maltrato junto con la supuesta victimización sexual, lo cual coincide con los estudios que señalan un riesgo incrementado de victimización sexual en niños objeto de negligencia (Finkelhor, More, Hanby y Straus, 1997) y en niños con antecedentes de maltrato físico (Boney-McCoy y Finkelhor, 1995).

El déficit psíquico hallado es próximo al registrado por Moreno et al. (1995) y por Sanmartín (2002) en niños víctimas de abuso. Ambos encuentran una mayor presencia de déficit psíquico en los niños victimas de abuso sexual, que en los de maltrato en general. Tal como afirman Gorman-Smith y Matson (1993) y Verdugo, (1993) la presencia del déficit psíquico en el niño podría ser considerado un factor de riesgo para el abuso sexual.

El absentismo escolar observado es similar al de otros niños víctimas de abuso sexual (Sanmartín, 2002) e inferior al de los niños víctimas de maltrato en general (Moreno et al. 1995; Sanmartín op. cit.). Este resultado plantea si el absentismo escolar no es tanto un factor de riesgo característico del abuso sexual, sino de cualquier tipo de maltrato.

Se confirma además la discreta presencia de indicadores físicos en niños de la muestra (Bays, Chadwidk, 1993), siendo mucho más frecuente la presencia conjunta de indicadores conductuales de alta y baja probabilidad.

\section{Caracteristicas de las familias}

Se ha encontrado una mayor representación del nivel socioeconómico bajo y precario, inclusive entre las familias de los niños no pertenecientes al sistema de protección, en la línea de investigaciones (Finkelhor 1997, Sdelak 1997, Paveza, 
1988) que encuentran que un nivel económico más bajo se relaciona con un mayor riesgo para el abuso sexual. El volumen de hacinamiento encontrado en la muestra informa que es menos frecuente su presencia entre las familias de niños supuestas víctimas de abuso sexual que en las de maltrato en general (Moreno et al. 1995; Sanmartín 2002) y es similar al hallado en otros niños supuestas víctimas de abuso sexual (Sanmartín, 2002).

En cuanto a la estructura familiar, se observa que predomina la estructura familiar compuesta por ambos progenitores. La presencia de familias monoparentales es ligeramente inferior a la encontrada en los casos de maltrato en general (Sanmartín, 2002), pero más elevada que la de la población andaluza general (Anuario estadístico de Andalucia, 2003). Casi la mitad de los niños de la muestra no convivían con su padre biológico cuando se produjo el abuso. Los anteriores resultados van en la línea de lo indicado por diferentes autores (Finkelhor 1997, Boney, McCoy y Finkelhor, 1995; Sedlak, 1997) con relación a que tanto la estructura familiar monoparental como el hecho de convivir con un solo padre biológico pueden ser factores de riesgo para el abuso sexual.

La mitad de las familias presentaron situaciones de violencia doméstica, en consonancia con los estudios, que han establecido relación entre la violencia doméstica en la familia y el riesgo de maltrato (Alexander, 1992; Deblinger, Hathaway, Lippmann, \& Steer, 1993; McCloskey, Figueredo, \& Koss, 1995; Suh, \& Abel, 1990) y el abuso sexual (Paveza, 1988).

En las madres se encontró una mayoría sin estudios o sólo con estudios primarios. Este dato no coincide con aquellos resultados (Finkelhor, 1997) que sostienen que el nivel educativo de los padres, no está en sí mismo relacionado con la victimización sexual. Respecto a la actividad laboral de las madres, los resultados se asemejan más a lo encontrado en otras investigaciones, (Jiménez, Oliva y Saldaña, 1996) en el sentido de que es más alto el porcentaje de madres activas entre las madres de niños víctimas de abuso sexual que entre las de maltrato. Se ha señalado que la ausencia prolongada del cuidador del hogar puede suponer un factor de riesgo para la victimización sexual (Finkelhor, 1997), los resultados apuntan en esta dirección, la mitad de las madres de la muestra pasaban mucho tiempo fuera del hogar. Otros autores (Dore, Doris, \& Wright, 1995; Famularo, Kinscherff, \& Fenton, 1992; Leifer, Shapiro, \& Kassem, 1993; Sheridan, 1995) han puesto de manifiesto la relación entre el abuso de sustancias por parte de los padres y el riesgo en los niños de padecer maltrato físico y abuso sexual. En nuestro estudio, el porcentaje de consumo de sustancias es similar al de madres de niños víctimas de abuso (Jiménez, Oliva, Saldaña, 1996), inferior al de las madres de maltrato en general (Moreno,1995) y superior a la población general. La presencia de déficit físico y psíquico encontrada es similar a lo hallado en el estudio de maltrato de Andalucía (Moreno, 1995) en los padres de niños víctimas de abuso sexual, pero muy superior al dato de la población general andaluza (Anuario Estadístico de Andalucia, 2003).

Finkelhor (1997) señaló que la victimización sexual parental previa está relacionada con un mayor riesgo de victimización sexual para los hijos de estas personas. Los resultados del presente estudio van en relativa consonancia con lo anterior, ya que lo más frecuente fue que las madres presentasen antecedentes de maltrato en general, desglosando el tipo 
de maltrato recibido, la mitad tenían antecedentes de abuso sexual.

Como señalan distintos autores (Everson, Hunter, Runyan, Edelsohn, \& Coulter, 1989; Heriot, 1996; Merrick, Allen, \& Crase, 1994), el apoyo al niño víctima es un importante factor para la recuperación, en la muestra sólo en la mitad de las parejas supuesta víctima-ofensor las madres mostraron una actitud de apoyo al niño al tener conocimiento del abuso.

\section{Caracteristicas de la sospecha de abuso}

Los resultados de la evaluación de las sospechas, se distribuyen en un continuo de confirmación o certeza del abuso, tal como es habitual en estas evaluaciones. El grado de mayor confirmación (alta probabilidad) se encuentra en cuatro de cada diez sospechas y el resto se distribuye en los restantes niveles. Estos datos coinciden con lo señalado por autores (Mraovich \& Wilson, 1999) que afirman que la valoración psicosocial del abuso sexual es limitada en sus conclusiones. No obstante, el número de sospechas 0 interacciones niño-ofensor diagnosticadas de alta probabilidad de ocurrencia fue similar a lo encontrado en otros estudios de centros específicos de evaluación de abuso sexual (Pou, 2002), pero sensiblemente inferior al volumen de diagnóstico de certeza que se señala en otros estudios (Moreno,1995). Sobre el testimonio de los niños, los resultados confirman lo expuesto por otros autores (Oates et al. 2000) en cuanto a la baja proporción de testimonios que son valorados como poco o nada veraces.

En consonancia, con el tipo de abuso más frecuentemente alegado en la muestra, el intrafamiliar, se observa que la duración del abuso predominante fue la del abuso largo o crónico. El siguiente más habitual fue el de corta duración. Hay ciertas similitudes con lo encontrado por otros autores (Pou, 2002).

En cuanto a la gravedad, cabe resaltar que casi la mitad fueron sospechas o interacciones niño-ofensor de carácter grave 0 muy grave, lo que matiza de modo sensible lo expuesto, en el sentido de que las características que se han ido enumerando, se refieren, para al menos la mitad de los casos, a situaciones de abuso sexual alegado graves o muy graves.

Con respecto al lugar dónde se producen los abusos, se coincide con otros estudios en la frecuencia citada para el domicilio del agresor, así cómo para el tipo de conducta ofensiva que esgrimieron los ofensores (Pou, 2002).

A modo de caracterización final, se puede presentar un perfil de los niños supuestas víctimas de abuso sexual estudiados según el cual predominan los niños, sobretodo las niñas, abusados en su mayoría por familiares; en número superior los que no pertenecian al sistema de protección, aunque con alta representación de los que sí pertenecían, por lo que no es de extrañar que en la misma proporción fueron objeto también de otros tipos de maltrato. Fueron más pequeños que en otros estudios, con abusos de carácter grave, con menos absentismo escolar que los niños víctimas de maltrato y con pocos indicios fisicos. Su abuso alegado consistió principalmente en tocamientos en genitales y muchos revelaron ser víctimas de abuso. En la mitad de sus familias se podía encontrar violencia doméstica, ausencia de padre biológico, madres que pasaban mucho tiempo fuera del hogar y que no apoyaron al niño cuando se desveló el abuso. 
Por último, y en cuanto a la comparación de los resultados con los de niños victimas de maltrato en general, señalar que hay resultados similares tanto en cuanto al tipo de abuso sexual predominante, que es el intrafamiliar, como en cuanto al nivel socioeconómico bajo y precario, el bajo nivel educativo de las madres, la presencia de violencia doméstica y el abuso de sustancias. Fueron diferentes en cuanto al hacinamiento, la actividad laboral en las madres, el género, el absentismo escolar y el déficit psiquico.

Las características de las supuestas víctimas más similares a las señaladas en otros estudios de niños víctimas de abuso sexual en nuestro contexto fueron: el tipo de abuso, el género, la presencia de otros maltratos en los casos de protección, el tipo de conducta abusiva y la baja presencia de indicios físicos.

Los datos encontrados sugieren la necesidad de analizar en futuros estudios cuáles de las variables descritas, podrian ser factores de riesgo comunes con el maltrato infantil y cuáles están específicamente ligadas al abuso sexual.

\section{BIBLIOGRAFÍA}

Anuario estadístico de Andalucía, 2003. Sevilla. Instituto de Estadística de Andalucía. Consejeria de Economía y Hacienda.

Alexander, P., C. (1992). Application of attachment theory to the study of sexual abuse. Journal of Consulting and Clinical Psychology, (60), 185195.

Bays, J,, y Chadwidk, D. (1993). Medical diagnosis of the sexually abused child. Child Abuse $y$ Neglect, (17), 91-110.

Bagely, C. y King, K. (1990). Child Sexual abuse: The search for healing. London: Routledge.

Berliner, L. y Elliott, D. M. (1996). Sexual abuse of the children. En J. Briere, L. Berliner, J. A. Bulkley, C. Jennit T. Reid (Eds.) The APSAC
Handbook on Child Maltreatment, (51-71). London: Sage Publication.

Boney-McCoy, S. y Finkelhor, D. (1995a). Prior victimization: A risk factor for child sexual abuse and for PTSD-related symptomatology among sexually abused youth. Child Abuse y Neglect, 19, (1), 1401-1421.

Boney-McCoy, S. y Finkelhor, D. (1995b). The psychosocial secuelae of violent victimization in a national youth sample. Journal of Consulting and Clinical Psychology, (63), 726-736.

Faller, K. C. (1993). Child sexual abuse: Intervention and treatment issues. The user manual series. Washington, DC: U.S. Department of Health and Human Services, Administration for Children and Families. 
De Jong, A. R., Hervada, A. R., y Emmett, G. A (1983). Epidemiologic variations in childhood sexual abuse. Child Abuse and Neglect, (7), 155162

De Paúl, J., Arruabarrena, M. I., Torres, D. y Muñoz, R. (1995). La prevalencia del maltrato infantil en la provincia de Guipozkoa. Infancia y aprendizaje, (71), 49-58.

Deblinger, E; Hathavay, C; Lippmann, J. y Steer, R. (1993). Psychosocial characteristics and correlates of sympton distress in nonoffending mothers of sexually abused children. Journal of Interpersonal Violence, (8), 155-168.

Dore, M. M., Doris, J.M. y Whight, P. (1995). Identifying substance abuse in maltreating families: A child welfare challenge. Child Abuse and Neglect, (19), 531-543.

Famularo, R., Kinscherff, R y Fenton, T. (1992). Parental Substance abuse and the nature of chil maltreatment. Child Abuse and Neglect, (16), 475483

Finkelhor, D. (1979). Sexually victimized children. New York: Free Press.

Finkelhor, D. (1986). A sourcebook on child sexual abuse. Beverly Hills: Sage Publications.

Finkelhor, D., Hotaling, G., Lewis, I. A., y Smith, C. (1990). Sexual Abuse in a national survey of adult men and women: prevalence, characteristics, and risk factors. Child Abuse and Neglect, (14), 19-28.

Finkelhor, D. (1993). Epidemiological factors in the clinical identification of child sexual abuse. Child Abuse and Neglect, (17), 67-70.

Finkelhor, D., More, D., Hanby, S., y Straus, M. (1997). Sexually abused children in a national survey of parents: methodological issues. Child Abuse y Neglect, 21 (X), 1-9.

Friedrick, W., Urquiza, A., y Bielke, R (1986) Behavior problems in sexually abused young children. Journal of Pediatric Psychology, 11 (1), 45-47.

Fischer, D. G. y McDonal, W. L. (1998). Characteristics of intrafamilial and extrafamilial child sexual abuse. Child Abuse y Neglect, 22, (9), 915929 .

Gorman Smith P. y Matson, J. L. (1993). Abuso sexual y personas con retraso mental. Siglo Cero, (146), 5-14.

Gracia, E., Musitu, G. (1993). El maltrato infantil. Un análisis ecológico de los factores de riesgo. Madrid: Ministerio de Asuntos Sociales.

Inglés, A. (1995). Origen, proceso y algunos resultados del estudio de malos tratos infantiles en Cataluña. Infancia y Aprendizaje, (71), 23-32.

Jiménez, J., Oliva A., Saldaña, D. (1996). Maltrato y protección a la infancia en España. Madrid: Ministerio de Asuntos Sociales.

Leifer, M.; Shapiro, J. y Kassem, L. (1993) The impact of maternal history and behavior upon foster placement and adjustment in sexually abused girls. Child Abuse and Neglect, 17 (6) 755-766

Leitenberg, H., Greenwald, E. y Cado, S. (1992). A retrospective study of long-term methods of coping with having been sexually abused during childhood. Child Abuse and Neglect, (16), 399407 .

Leventhal J. M. (1998) Epidemiology of sexual abuse of children: old problems, new directions. Child Abuse y Neglect, 22, (6), 481-491.

López, F., Hernandez, A. y Carpintero, E. (1995). Los abusos sexuales de menores: concepto, prevalencia y efectos. Infancia y Aprendizaje, (71), 77-98.

López, F. (1994). Los abusos sexuales de menores: Lo que recuerdan los adultos. Madrid . Ministerio de Asuntos Sociales.

McCloskey, L.; Figueredo, A.; Koss, H. (1995). The effects of systemic family violence on children's mental health. Child Development, 66 (5), 12391261.

Mannon K., Leitschuh G. (2002). Child Sexual Abuse: A Review of definitions, Instrumentation, and Symptomology. North American Journal of Psychology, 14, (1), 149-160.

Mian, M., Wehrspann, W., Klajner-Diamond, H., LeBaron, D., y Winder (1986). Review of 125 children six year of age and under who were sexually abused. Child Abuse y Neglect, (10), 223-229.

Moreno, M.C., Jiménez, J., Oliva, A., Palacios, J., y Saldaña, D. (1995). Maltrato infantil en Andalucía. Sevilla: Consejería de Asuntos Sociales de la Junta de Andalucía. 
Oates R.K., Jones D., Denson D., Sirotnak, A., Gary N., y Krugman, R.D. (2000). Erroneus concerns about child sexual abuse. Child Abuse y Neglect, 24, (1), 149-157.

Paredes M., Leifer M, y Kilbane T. (2001). Maternal variables related to sexually abused children's functioning. Child Abuse y Neglect, (25), 1159-1176.

Paveza, G. J. (1988). Risk factors in fatherdaughter child sexual abuse. A case control study. Journal Interpersonal Violence, (3), 290-306.

Pou, J., Jordan I., Gómez L. (1996). Abuso sexual infantil. Nuestra experiencia de un año. Anales Españoles de Pediatría, (45), 245-248.

Pou, J., Ruiz, A., Comas, Ll., Petitbó M. D., Ibáñez, M. y Bassets, J. (2001). Abuso sexual infantil: experiencia de una unidad funcional a menores. Anales Españoles de Pediatría, (54), 243-250.

Pou, J., Comas, Ll., Petitbó, M. D., Ibáñez, M. y Macias C. (2002). Abuso sexual. Experiencia en una unidad funcional de abusos a menores. Bienestar y Protección Infantil, I, (1),57-69.

Rind, B., Tromovitch, P., y Bauserman, R. (1998). A meta-analytic examination of assumed properties of child sexual abuse using college samples. Psychological Bulletin, (124), 22-53.

Sanmartin, J. (2002). Maltrato infantil en la familia. España 1997-1998. Valencia: Centro Reina Sofia para el Estudio de la Violencia.

Sedlak, K. A. (1997). Risk factors for the occurrence of child abuse and neglect. Journal Aggression Maltreatment Trauma, 1 (1), 149-187).

Schaaf, K., y McCanne, T. (1998). Relationship of childhood sexual, physical and combined sexual and physical abuse to adult victimization and posttraumatic stress disorder. Child Abuse and Neglect, 22 (11), 1119-1133.

Suh, E. y Abel, E., m. (1990). The impact of spousal violence on the children of the abused. Journal of Independent Social Work, 4 (4), 27-34.

Verdugo, M. A., Gutiérrez, B., Fuertes, J., Elices, J.A., (1993). Maltrato infantil y minusvalía Madrid: Ministerio de Asuntos Sociales.

Tseng, O.C. y Schwarcyn, H.J. (1990). Gender and races differences in child sexual abuse correlates. International Journal of Intercultural Relations, (14), 135-161. 